\title{
Type I interferon in human autoimmunity
}

\author{
Timothy B. Niewold * \\ Division of Rheumatology and Department of Immunology, Mayo Clinic, Rochester, MN, USA \\ ${ }^{*}$ Correspondence: niewold.timothy@mayo.edu \\ Edited by: \\ Claudia Kemper, King's College London, UK \\ Reviewed by: \\ Giovanna Schiavoni, Istituto Superiore di Sanità, Italy
}

Keywords: interferons, systemic lupus erythematosus, Sjogren's syndrome, multiple sclerosis, scleroderma, systemic, type I diabetes, autoimmune thyroid disease

The type I interferon system plays a critical role in host defense in health, and a growing body of literature suggests that type I interferon is a critical mediator of human autoimmune disease (1). Type I interferons function as a bridge between the innate and adaptive immune systems, and as such play an important role in setting thresholds for response against self antigens. Many investigators have focused on the role type I interferons play in autoimmune disease. This fascinating and rapidly growing body of literature encompasses many different autoimmune diseases, including systemic lupus erythematosus, type I diabetes, multiple sclerosis, and others. Type I interferons play differing roles in human autoimmune conditions. For example, in the autoimmune diseases, systemic lupus erythematosus and Sjogren's syndrome, increased interferon alpha signaling plays a pathogenic role (2, $3)$. Interestingly, interferon beta is used as a therapeutic in multiple sclerosis, an autoimmune disease of the central nervous system (4). Both interferon alpha and beta signal through the same type I interferon receptor and share many similarities in downstream signaling, suggesting that the disparate activities of type I interferons in lupus and multiple sclerosis relate to differences in the underlying disease processes and immunoregulation in these two diseases. In this Research Topic, a series of articles provides a comprehensive overview of the various roles type I interferons play in autoimmune diseases, with a focus on human immunology.

This Research Topic features a number of Original Research Articles, including a study by Mavragani et al. examining type I interferon levels in the organ-specific autoimmune disorders type I diabetes and autoimmune thyroid disease (5). They demonstrate high type I interferon levels in both of these autoimmune conditions, supporting the idea that high levels of type I interferon are detectable in organ-specific autoimmune conditions in addition to systemic autoimmune disorders. Clark et al. investigate genetic polymorphisms in the interferon regulatory factor 5 (IRF5) gene (6). This gene has been associated with susceptibility to systemic lupus erythematosus (7), and they demonstrate four distinct promoter regions have differential activity. Ko et al. study type I interferon-induced gene expression in patients with systemic lupus erythematosus (8). They demonstrate that the expression of type I interferon-induced genes in lupus immune cells differs significantly between ancestral backgrounds, which corresponds to clinical differences in the disease between ancestral backgrounds. A Methods article by Feng et al. examines public domain gene expression data to document patterns of type I interferon-induced gene expression and infer both positive and negative regulation by transcription factors (9).

The Research Topic also features a number of Review Articles focusing on various disease states. Liu et al. review murine models of systemic lupus erythematosus that are interferon-inducible, providing model systems of autoimmunity related to type I interferon (10). Wu et al. review the role of type I interferon in systemic sclerosis, a distinct autoimmune disease characterized by thickening and fibrosis of the skin, which shares a type I interferon signature with other autoimmune conditions (11). Li et al. review the evidence supporting a role for type I interferon in the pathogenesis of Sjogren's syndrome, spanning genetic associations, gene expression studies, and clinical features of the disease (12). Reder et al. review the contrasting role of type I interferon in multiple sclerosis and systemic lupus erythematosus and other autoimmune conditions (13). In multiple sclerosis, type I interferon levels are low (14), and administration of recombinant type I interferon is an effective treatment. They review the evidence supporting multiple sclerosis as a low interferon autoimmune disease, and speculate on immunological features that might underlie this striking difference. Shrivastav et al. review the role of nucleic acid receptors in type I interferon generation in systemic lupus erythematosus (15), a disease characterized by pathological activation of the type I interferon pathway. These articles taken together provide an overview of many of the ways type I interferons have been implicated in human autoimmune disease, providing a fascinating window into the biology of the human immune system gone wrong.

\section{ACKNOWLEDGMENTS}

Funding sources: Timothy B. Niewold - research grants from the NIH (AR060861, AI071651), Lupus Foundation of Minnesota, Mayo Clinic Foundation, and the Rheumatology Research Foundation.

\section{REFERENCES}

1. Ghodke-Puranik Y, Niewold TB. Genetics of the type I interferon pathway in systemic lupus erythematosus. Int J Clin Rheumtol (2013) 8:657-69. doi:10.2217/ijr.13.58

2. Emamian ES, Leon JM, Lessard CJ, Grandits M, Baechler EC, Gaffney PM, et al. Peripheral blood gene expression profiling in Sjogren's syndrome. Genes Immun (2009) 10:285-96. doi:10.1038/gene.2009.20 
3. Ko K, Franek BS, Marion M, Kaufman KM, Langefeld CD, Harley JB, et al. Genetic ancestry, serum interferon-alpha activity, and autoantibodies in systemic lupus erythematosus. J Rheumatol (2012) 39:1238-40. doi:10.3899/ jrheum.111467

4. Javed A, Reder AT. Therapeutic role of beta-interferons in multiple sclerosis. Pharmacol Ther (2006) 110:35-56. doi:10.1016/j.pharmthera.2005.08. 011

5. Mavragani CP, Niewold TB, Chatzigeorgiou A, Danielides S, Thomas D, Kirou $\mathrm{KA}$, et al. Increased serum type I interferon activity in organ-specific autoimmune disorders: clinical, imaging, and serological associations. Front Immunol (2013) 4:238. doi:10.3389/fimmu.2013.00238

6. Clark DN, Read RD, Mayhew V, Petersen SC, Argueta LB, Stutz LA, et al. Four promoters of IRF5 respond distinctly to stimuli and are affected by autoimmunerisk polymorphisms. Front Immunol (2013) 4:360. doi:10.3389/fimmu.2013. 00360

7. Cham CM, Ko K, Niewold TB. Interferon regulatory factor 5 in the pathogenesis of systemic lupus erythematosus. Clin Dev Immunol (2012) 2012:780436. doi:10.1155/2012/780436

8. Ko K, Koldobskaya Y, Rosenzweig E, Niewold TB. Activation of the interferon pathway is dependent upon autoantibodies in African-American SLE patients, but not in European-American SLE patients. Front Immunol (2013) 4:309. doi:10.3389/fimmu.2013.00309

9. Feng D, Barnes BJ. Bioinformatics analysis of the factors controlling type I IFN gene expression in autoimmune disease and virus-induced immunity. Front Immunol (2013) 4:291. doi:10.3389/fimmu.2013.00291

10. Liu Z, Davidson A. IFNalpha inducible models of murine SLE. Front Immunol (2013) 4:306. doi:10.3389/fimmu.2013.00306

11. Wu M, Assassi S. The role of type 1 interferon in systemic sclerosis. Front Immunol (2013) 4:266. doi:10.3389/fimmu.2013.00266
12. Li H, Ice JA, Lessard CJ, Sivils KL. Interferons in Sjogren's syndrome: genes, mechanisms, and effects. Front Immunol (2013) 4:290. doi:10.3389/fimmu.2013. 00290

13. Reder AT, Feng X. Aberrant type I interferon regulation in autoimmunity: opposite directions in MS and SLE, shaped by evolution and body ecology. Front Immunol (2013) 4:281. doi:10.3389/fimmu.2013.00281

14. Feng X, Reder NP, Yanamandala M, Hill A, Franek BS, Niewold TB, et al. Type I interferon signature is high in lupus and neuromyelitis optica but low in multiple sclerosis. J Neurol Sci (2012) 313:48-53. doi:10.1016/j.jns.2011. 09.032

15. Shrivastav M, Niewold TB. Nucleic acid sensors and type I interferon production in systemic lupus erythematosus. Front Immunol (2013) 4:319. doi:10.3389/ fimmu.2013.00319

Conflict of Interest Statement: The author declares that the research was conducted in the absence of any commercial or financial relationships that could be construed as a potential conflict of interest.

Received: 08 June 2014; accepted: 16 June 2014; published online: 30 June 2014.

Citation: Niewold TB (2014) Type I interferon in human autoimmunity. Front. Immunol. 5:306. doi: 10.3389/fimmu.2014.00306

This article was submitted to Molecular Innate Immunity, a section of the journal Frontiers in Immunology.

Copyright (C) 2014 Niewold. This is an open-access article distributed under the terms of the Creative Commons Attribution License (CC BY). The use, distribution or reproduction in other forums is permitted, provided the original author(s) or licensor are credited and that the original publication in this journal is cited, in accordance with accepted academic practice. No use, distribution or reproduction is permitted which does not comply with these terms. 